\title{
Standardized Communication in the Control System of the Experiment WENDELSTEIN 7-X
}

\author{
J. Schacht, H. Laqua, M. Lewerentz, A. Spring, S. Pingel, G. Kühner
}

\begin{abstract}
The super conducting stellarator experiment W7-X has a control system which has been designed for steady state operation as well as for pulsed operation. Each technical component and each "diagnostic" system including its data acquisition will have its own control system permitting autonomous operation for commissioning and testing. During the experimental sessions the activity of these components will be coordinated by a Central Control System and the machine runs more or less automatically with predefined programs. A local control component has a number of internal and external communication interfaces which are necessary for data exchange with the operational management system, the segment control system and with the safety system. These interfaces are used to send and receive messages of different types (commands, status information, raw data, and analyzed data). The paper presents a description of the structure of a local control component and a discussion of its communication interfaces.
\end{abstract}

Index Terms - control system, distributed real time system, fusion experiment, messages, standardization of messages.

\section{INTRODUCTION}

$\mathrm{T}$ HE fusion experiment Wendelstein 7- X (W7-X) is a large superconducting stellarator project under construction in Greifswald, Mecklenburg-Vorpommern, Germany. The main goal of $\mathrm{W} 7-\mathrm{X}$ is to demonstrate the conceptual reactor capability of stellarators including the steady state operation.

The physics goal of W7-X demands a new concept for the control system of this experimental device. The control system is able to process different scenarios of the discharge operation. These scenarios can be classified into three main types: short pulses of a few second duration, long pulses with arbitrary sequences of short phases with different objectives and device configuration, and steady state operations in which a certain plasma state is being kept for unlimited time (Fig.1). Steady state operation can be planned with durations up to 30 min with full Electron Cyclotron Resonance (ECRH) -heating power or even longer with lower power.

Experimental runs are divided into phases called segments.

Manuscript received April 20, 2007; revised December 3, 2007.

J. Schacht, H. Laqua, M. Lewerentz, A. Spring, S. Pingel, and G. Kühner: Max-Planck Institute für Plasmaphysik, Teilinstitut Greifswald, D-17489 Greifswald, Germany. (e-mail: joerg.schacht@ipp.mpg.de).
Each segment describes the behaviour of all technical and diagnostic components involved in the experiment operation.

Thus the planned plasma discharge consists of a sequence of predefined segments to be carried out in real time.

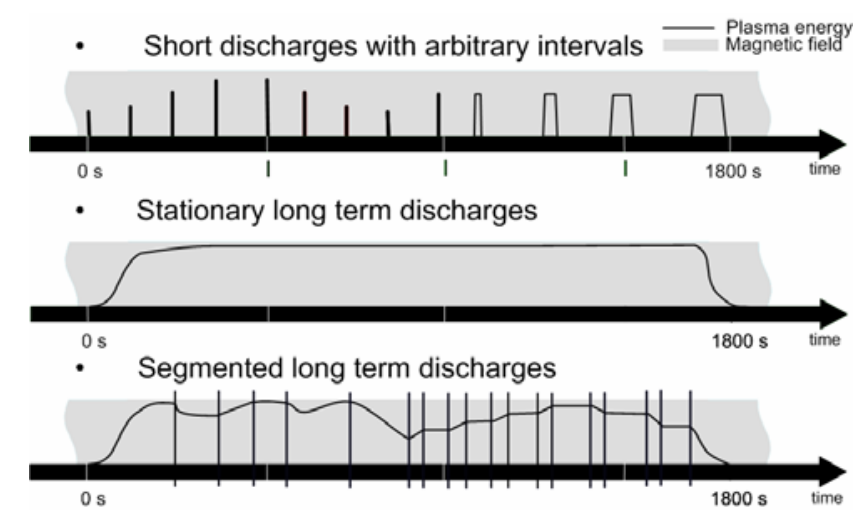

Fig. 1. Segmentation types of the Wendelstein 7-X discharges

The segment wise description of the device control behavior provides maximum flexibility in plasma operation. A segment sequence controller transmits so called segment switch messages in order to trigger all control computers to synchronously process the segments within the experiment program.

\section{BUSINESS MODEL FOR THE W7-X CONTROL SYSTEM}

A simplified business model for the main processes of the W7-X control system is shown in Fig. 2.

This model consists of 5 main parts, necessary for planning, preparation and execution of experiment programs with a fusion device: preparation and interaction, control, data acquisition and processing, data visualization and storage, as well as safety protection. Every model part includes sub parts for a detailed description of model behaviour and processes. The model parts have connections to other parts for exchange of data, signals and commands.

The control system is designed for active interaction and control of a large number and a wide spectrum of physical and technical processes in the phases of experiment preparation and execution. The model part "Control processes" gives a description of the behaviour of the processes. For interaction with the processes the control processes uses actors, e.g. the 
actor for the control process "Setup the magnetic configuration of $W 7-X$ ” are the magnet power supplies for the superconducting coil system. prediction of plasma states and for an assessment of the models in use.

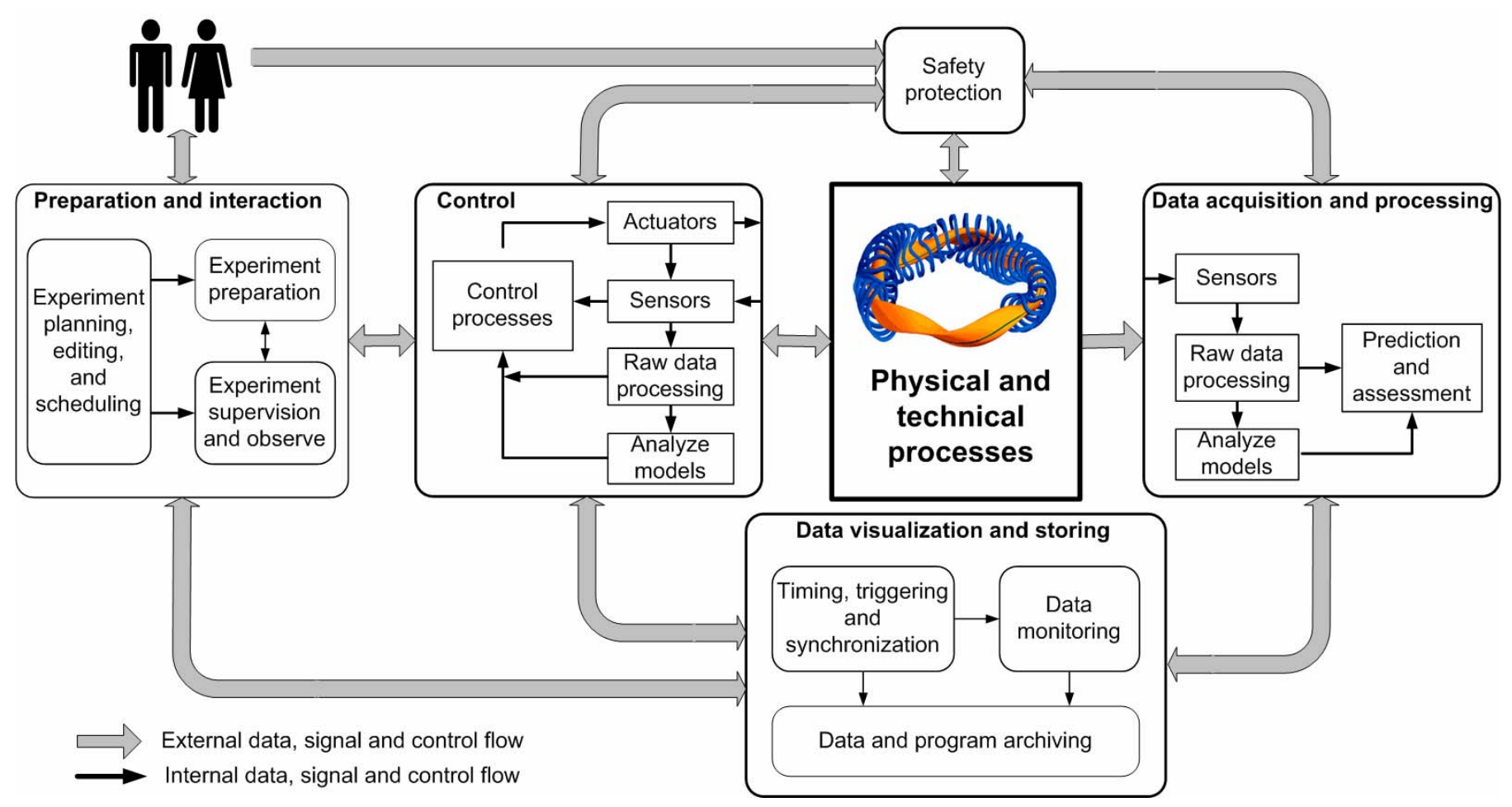

Fig. 2. Business model for Wendelstein 7-X control system

The control process needs information about the actual state of the actors and of the controlled process itself. This sort of information is produced by a set of sensors, which generates status information or raw data of technical or physical processes. In many cases these measured values are input values for signal processing, e.g. calculation of characteristic statistical values, signal filtering and so on. The business model summarized all these activities in the sub model "Raw data processing". The sub model "Analysis model” describes all processes of calculation of high level status values such as density profiles of the plasma by using analytical physics models. As the analysis models are designed to calculate actual process control values, their results directly influence the control processes in real time. All control processes have to be prepared, controlled and observed. If necessary, an interaction on processes can be established by the user (experiment engineers, physicist).

Acquisition of scientific data from experiments and their processing is encapsulated in the sub model "Data acquisition and processing”. Differing from the sub model "Control" the data acquisition is focused on measurements of plasma physical data for characterization of plasma status. Basically, the measurements are made by different diagnostic systems, which produce data streams with high data rates and different data types (e.g. profiles, images, and event statistics). The raw data, and data of the analysis models, can be used for a
The sub models control, data acquisition, as well as preparation and interaction are closely connected to the sub model "Data visualization and storing”.

All experiment program information, parameter settings of the control and data acquisition systems, the experiment status information and the experiment results have to be stored in an archive. Furthermore, data have to be distributed for monitoring and visualization purposes.

The sub model "Preparation and interactions" comprises planning, editing and scheduling of W7-X experiment programs. Also functions for preparation, supervision, and observation of the experiments are described by this sub model.

The machine W7-X has a high potential of different hazards. Personnel near the device may be injured and equipment may be damaged by mechanical, electrical, magnetic, thermal impact, by radiation or oxygen deficiency which all have to be prevented by a safety control system. All safety relevant operations are summarized into the sub model "Safety protection".

As shown in Fig. 2 the sub models of the business model have a wide amount of internal and external data, signal and control flows.

On the base of this business model of the W7-X control system the main processes of the operation and its requirements have been identified. 
In the implementation phase of the W7-X control system all model parts of the business model must be implemented as parts of central or local control components.

\section{IMPLEMENTATION OF W7-X CONTROL SYSTEM}

The W7-X control system has a hierarchical structure, consisting of the central control system on the top and a large number of local control components at the bottom. The local control components are designed to fulfil special purposes, e.g. plasma heating, cooling or diagnostic purposes. A local control component can be a technical component or a diagnostic system including its data acquisition system. An integrated approach has been employed to develop a standard description for the basic structure of a local control component (see Fig.3). This allows a faster design of individual component control systems by reuse of proven modules and patterns.

A component can be equipped with different types of controllers. PLCs (Programmable Logic Controller) are used for slow control processes and for the operational management. The operational management system is divided into the operational management of the components (local OPM: LOPM) and the central operational management of the device W7-X (central OPM: COPM). The main tasks of the operational management are switching of the operational states of W7-X and its components and a visualization of important status and process information.

The segment control system is responsible for the preparation, processing, and monitoring of the predefined and validated experiment programs into the control station (Fast control stations and DAQ stations). Control processes with real time requirement are accomplished by standard PCs (FC stations: Fast Control station) using a real time operating system (OS). Data acquisition tasks with high data rates, an integrated raw data processing, and minor control tasks are implemented on standard PCs (CODA station: Control and Data Acquisition station), equipped with a standard OS (e.g. Linux or Windows XP) and special measurement hardware. The behaviour of the component FC and DAQ stations is adjusted by the definitions of the segment description of the experiment program.

This standard component model leads to a standardization of data and signal exchange between component control modules and components themselves, too.

The local component permits autonomous operation for commissioning and testing.

During experiment operation all necessary local components are subordinated and all activities of these control systems are coordinated by the central control systems. The coordinated cooperation of several groups (local control components) is described as a project. In the W7-X data model a group corresponds with a component.

Fig. 3 illustrates the communication in the W7-X control system hierarchy. A large number of interfaces are installed to exchange control instructions, data, time, and synchronization signals. A standard network, based on the TCP/IP protocol is used for transmitting all information without or soft real time requirements. The real time network, physically separated from the standard network, is used for the distribution of event messages and measured values with a requirement of delivery within less than $100 \mu$. Time and event messages with harder real time requirements are distributed via a fiber optical network with a guaranteed packet delivery within $10 \mu$ s [1] [2].

The central sequence control system processes the predefined experiment programs and the machine will run more or less automatically.

A session leader program is used as a Human Machine Interface (HMI) between leader of the experiment and the central sequence control. It allows to choose and to chain predefined segments as experiment programs, and to execute and manipulate the program.

A fully detailed description of the tasks and structure of the W7-X control system is given in [3] [4] [5] [6] [7] [8] [9].

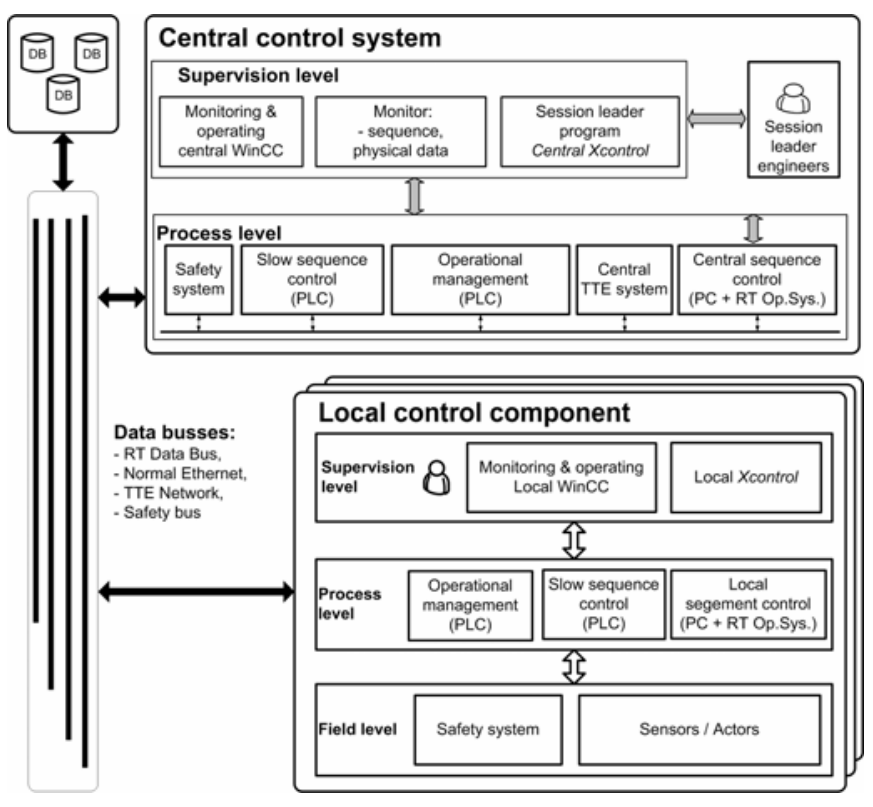

Fig. 3: Overview W7-X control system

\section{Control System MEsSAges}

The W7-X control system is a distributed system, which consists of more than 50 technical, infrastructure and diagnostic control components for the first operational phase after commissioning. The partition of the control tasks into the main areas of operational management and segment control and a uniform component structure facilitates the definition of standard control functions and of their communication. Each control component is designed to fulfill control and data acquisition tasks concerning its own group. This requirement has to be considered in the definition of standard messages.

\section{A. Message Classification}

Basically, the messages for W7-X control and data acquisition purposes can be divided into two types with 
respect to their time behavior: Messages can be sent nonperiodically or periodically (see Fig. 4). The group of nonperiodical messages involves the messages of type Notification and Event.

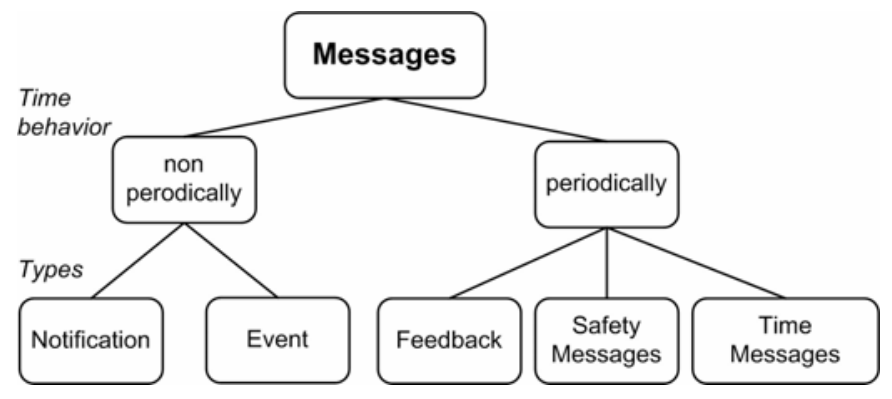

Fig. 4. Message classification for W7-X control system

\section{(1) Notification Messages}

Notification messages are used by the control stations of the components for sending status and fault diagnostics. A Notification server gathers all notification messages sent by the distributed units. The messages are stored in a data base from where they can be retrieved for later evaluation. The notification messages can also be used for monitoring purposes. There are three subtypes of notification messages: status messages, warnings and errors. All notification messages are sent as UDP (user datagram protocol) multicast data packets via the standard Ethernet. The format of the message is XML (extensible markup language). The parameter notificationThreshold of the transmitter determines, which types of notifications have to be sent (e.g. $0=$ send all notifications, $3=$ send only fatal errors). The structure of notification messages is as follows:

- Severity level of the message: debug info, info, warning, error,

- Time: time of creation of the message,

- Text: description of the event, which caused the message,

- Name CS: name of the sending control station,

- Location: Source code name and raw number of the producing message,

- Task name: name of the task, which has caused the message.

\section{(2) Event Messages}

Messages of the type Event are used for the distribution of control events and plasma-physics events. The source of this type of message can be a local component's control station or the central control system. A control station can configure individually the behavior on receiving a message. The responses are defined by so called actions, which are started after a dedicated message occurred. The segment description provides the possibility to define the reaction of a message for each segment.

The structure of the Event messages is as follows:
- ID (Identifier): Each message is assigned to a message category by a number in the range of $0-1023$,

- Length: Value for the length of the data packet.

- CRC: Cyclic Redundancy Checksum (CRC) calculated over the user data.

- Data packet: Each message contains a data packet, which is specific for the ID. The structure of the data can be set by using basic data types (e.g. int, float, array[], string,...). For every message a class has to be defined with the necessary data structure and methods to serialize and deserialize the message. The data are serialized in network byte order (big endian) in analogy to the XDR protocol (external data representation, RFC 1832).

The transport layer for sending messages of type Event can be:

- Ethernet Layer 2 with type 0xC001 MAC multicast,

- Ethernet Raw IP unicast or multicast,

- Trigger Time Event network (TTE network); only for Event messages.

The use of multicast messages allows a filtering of the incoming data stream, e.g. by the hardware filter of the network interface. Only multicast messages with an address for which the filter of the interface has been set properly can be received and processed.

\section{(3) Feedback Messages}

Messages of type Feedback are transferred periodically. The time period for a feedback message is set individually during the configuration. The structure of the Feedback messages is identical to the Event message. All transport layers used for Events except for the TTE network can be used for Feedback messages, too. If the sending of Feedback messages is triggered by the control station's timing system, the processing of this type of message can be performed synchronously in different control stations.

\section{(4) Time Messages}

The central Trigger Time Event system (TTE system) consists of two identical subsystems (master and slave timing system) for redundancy reasons. Only the active master is enabled to send out time messages. Time messages are distributed periodically every $100 \mathrm{~ms}$ from the hardware timer system of the central TTE system to the component's control stations via a unidirectional fiber optic network. The time message has a fixed data structure and consists of following parts:

- Identifier “Time Mark” (8 bit),

- Value of the central time counter (64 bit),

- Name of the sending timing system (name of the active master timing system),

- Status information of the central timing system.

\section{(5) Safety Messages}

Safety related states of the whole W7-X device and of the 
control components are distributed via a safety network running fail-safe Profibus. An interface close to the component's control systems transforms the safety related messages into electrical signals. The safety messages are status information and commands. The standard interface transfers the signals:

- Command: Emergency stop signal (central safety system $\rightarrow$ component),

- Status: Component is in emergency stop state (component $\rightarrow$ central safety system),

- Status: Component is in a safe state (component $\rightarrow$ central safety system),

- Status: Enable signals for hazardous activities of the component (central safety system $\rightarrow$ component),

- Status: Failure $x y$ is detected (central safety system $\rightarrow$ component).

\section{B. Standardized Control Messages}

A standardization of messages has widely been established for data and command exchange between components of the operational management system and the segment control system (see Fig. 5).

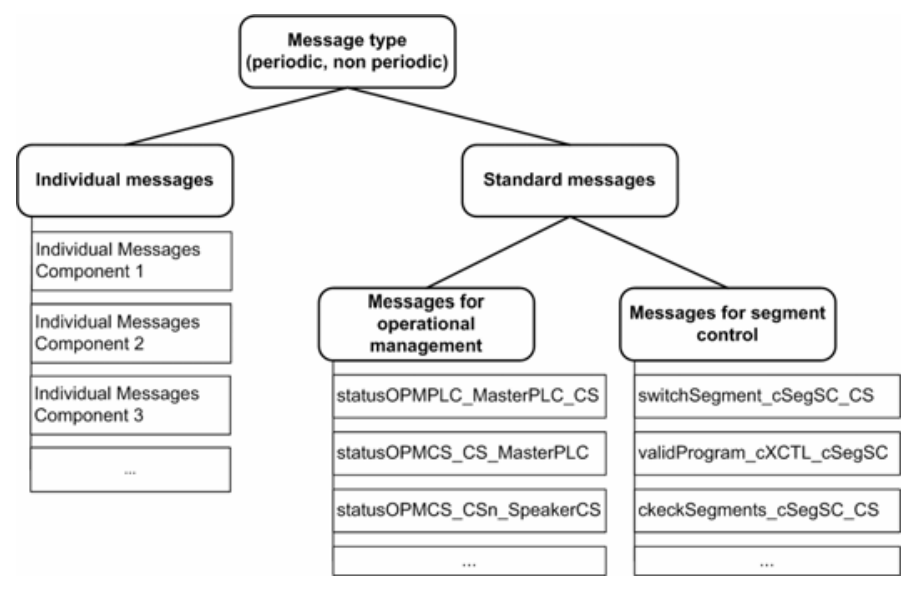

Fig. 5. W7-X messages types

\section{Message <statusOPM_MasterPLCXxx_cOPM>}

\section{Standard part}

- Identification,

- Status "Authority to control“

- Status „Communication watch dog“,

- Standard signals component status: Operational modes: ON, OFF, PREPARATION,...

- Extended standard signals:

Operational modes, enable signals, events of faults,

Producer specific part

- Component specific information: status information,

set values,

actual values,

limit value,...

Fig. 6. Structure of a message at the example message

statusOPM_MasterPLCxxx_cOPM

Some of the control messages are identical for all control components of W7-X. In order to increase the grade of message standardization the message structure shown in Fig.6 has been introduced. All parts of a message type, which are identical for all components, are summarized in the standard part of the message. In the second part the component specific information of a given message type is located. The advantages of this message structure are besides a standardization of more control messages a better efficiency of the software implementation.

The name scheme for a message consists of three parts:

1. Message name: <short symbolic name $>$,

2. Message producer: $<$ source $>$,

3. Message receiver: < destination>.

Multicast messages use only the first two parts for its message name.

\section{Examples For_Processing Of Standard Messages}

\section{(1) StatusOPM Message}

The master PLC of each component collects information about the operational state from all controllers of the component (slave PLCs, control stations). For example, every control station sends its own status message statusOPM_CS\#n_Speaker_CS. The 'speaker' of a component's control stations receives all control stations' status messages and builds with this information a summarized status information about the operational states of the controllers.

The message statusOPMCS_speaker_CS_MasterPLC contains this status information and is sent to the Master PLC. The status information of the controllers and of the slave PLC is combined for the operational status message of the component.

The message statusOPMComponentXY_IOPM_cOPM have to be sent to the PLC of the central control system (COPM), responsible for operational management of the whole W7-X device. The COPM analyzes these status messages and visualizes them.

Fig. 7 shows the data flow necessary for building of the operational status of a project. The distribution of standard messages is organized in such a way, that a unicast protocol is used for messages with one dedicated receiver (e.g. message statusOPMCSn_CS\#n_Speaker_CS). A Multicast protocol is used for messages with more than one receiver (e.g. message statusOPMProjectW7-X_cOPM).

The device status can be built periodically (in a range of 1-5 $\mathrm{Hz}$ ) by using the status information of the components. The message statusOPMProjectW7-X_cOPM will be transmitted via multicast and can be received by all control components, if necessary. 

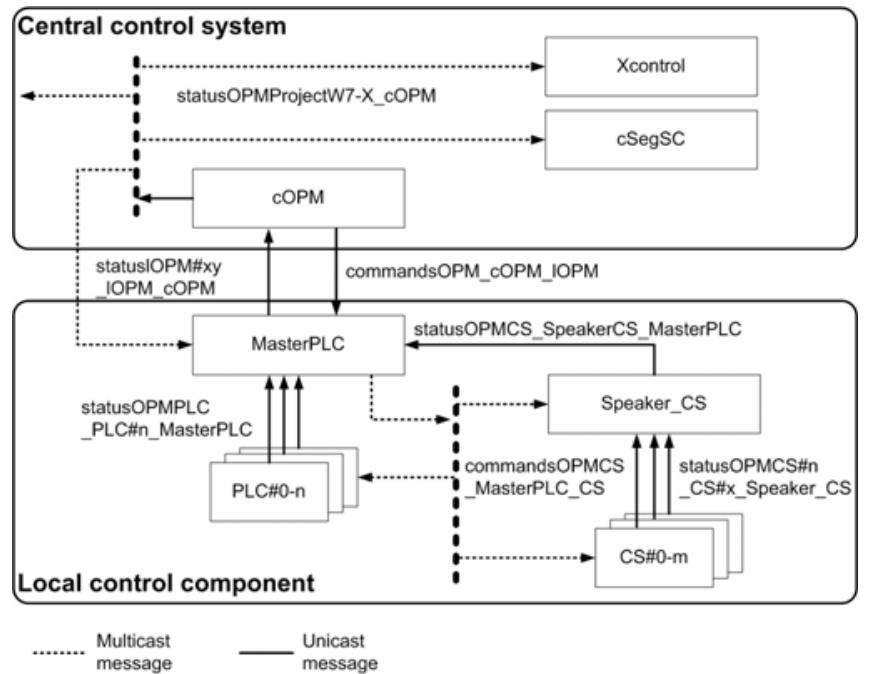

Fig. 7. Message flow for determining of the actual operational status of a component

Commands of the operational management (e.g. switch of the operational state, new set points) are distributed form the Master PLC via multicast to all control stations and PLCs of the component. Fig. 8 gives an overview of the structure of the message statusOPMProjectW7-X_cOPM. The definition of message data structures has been done as $C++$ or Java class for the control stations and as so called Data Modules for the PLCs. The configuration of a control station contains parameters for the configuration of control messages of types Event and Feedback. The configuration can be edited by using the special configuration and segment program editor “ConfiX” [10] [11] [12].

\begin{tabular}{|c|c|c|}
\hline \multicolumn{3}{|c|}{ Message <statusOPMProjectW7-X_cOPM> } \\
\hline $\begin{array}{l}\text { Standard part } \\
\text { - Identification: } \\
\text { - Machine state: } \\
\text { - } \\
\text { - } \\
\text { - } \\
\text { - N objects: } \\
\text { - Operational states } \\
\text { of all project groups: }\end{array}$ & $\begin{array}{l}\text { 0x4040 (Project W7-X) } \\
\text { 0: Emergency stop } \\
\text { 1: OFF State } \\
\text { 2: Stand By } \\
\text { 3: Experiment pause } \\
\text { 4: Experiment operation } \\
\text { 5: Partly protecded mode } \\
\text { (e.g. W7-X, WEGA) } \\
\text { number of objects in pairlist } \\
\text { op. state of component xy } \\
\text { (part of statusOPMxy_IOPM_cOPM) }\end{array}$ & $\begin{array}{l}32 \text { Bit integer } \\
\text { pairs of } \\
32 \text { Bit integer }\end{array}$ \\
\hline $\begin{array}{l}\text { Producer specific } \\
\text { - Empty }\end{array}$ & & \\
\hline
\end{tabular}

Fig. 8: Structure of a status operational management message sent by the PLC of the central operational management of project W7-X

\section{(2) SegmentSwitch Message}

The message segmentSwitch is an example for an asynchronous segment control message. It is a command transmitted from the central segment sequence controller (cSegSC). Using the multicast protocol all FC and CODA stations of the $\mathrm{W} 7-\mathrm{X}$ device receive this message. This message contains the identification of the next segment description. The FC and CODA stations activate the new segment at the given point in time. Fig. 9 shows the structure of the message. It has only a standard part. The message consists of an identification of the message type, identification for the project, for which is the message valid, a number for the new segment description and the time value for the absolute transmitting time of the switch message.

\begin{tabular}{|c|c|c|}
\hline \multicolumn{3}{|c|}{ Message <SwitchSegment_cSegSC $>$} \\
\hline $\begin{array}{l}\text { Standard part } \\
\text { - Identification: } \\
\text { - Function group ID: } \\
\text { - Segment number: } \\
\text { - Time stamp: }\end{array}$ & $\begin{array}{l}\text { 0x8001(Project W7-X), } \\
\text { Identification of Project } \\
\text { (e.g. W7-X, WEGA) } \\
\text { Switch to segment } x x \\
\text { Absolute time to switch }\end{array}$ & $\begin{array}{l}16 \text { Bit integer } \\
32 \text { Bit integer } \\
32 \text { Bit integer } \\
64 \text { Bit integer }\end{array}$ \\
\hline $\begin{array}{l}\text { Producer specifi } \\
\text { - Empty }\end{array}$ & & \\
\hline
\end{tabular}

Fig. 9: Structure of a segment switch message

\section{ACKNOWLEDGEMENTS}

The authors would like to thank the data acquisition group and the W7-X software group for their support and helpful discussions.

\section{REFERENCES}

[1] J. Schacht, H. Niedermeyer, H. Laqua, "The Trigger-Time-Event System for the W7-X Experiment”, Proceedings of the 12th IEEE Real Time Conference on nuclear and plasma science, Valencia, June 2001, S.240244.

[2] J. Schacht, H. Laqua, H. Niedermeyer, "Synchronization of processes in a distributed real time system exemplified by the control system of the fusion experiment WENDELSTEIN 7-X, Proceedings of the $14^{\text {th }}$ IEEE Real Time Conference on nuclear and plasma science, Stockholm, May 2005,

[3] H. Laqua, H. Niedermeyer, J. Schacht, “Control System of Wendelstein 7-X Experiment”, Fusion Engineering and Design 66-68, 669-673, 2003

[4] J. Schacht, H. Niedermeyer, J. Hildebrandt, Chr. Wiencke: The TriggerTime-Event System for the W7-X Experiment, 3 rd. IAEA Technical Committee Meeting on Control, Data Acquisition and Remote Participation for Fusion Research, Padova 2001

[5] J. Schacht, H. Laqua, A. Spring et al., "Task and structure of the WENDELSTEIN 7-X control system”, Fusion Engineering and Design 81 (2006), 1799-1806

[6] A. Werner, A. Dinklage, G. Kühner et al., "Integrated Software Development for Wendelstein 7-X", Proceedings IAEA Fusion Engineering FT/P7-6, Chengdu, 2006

[7] J. Schacht, H. Niedermeyer, H. Laqua. et al., Tasks and structure of the WENDELSTEIN 7-X control system, Fusion Eng. Design 81 (2006), 1799

[8] A. Spring, H. Laqua, H. Niedermeyer, "User interaction concept for plasma discharge control on WENDELSTEIN”, Fusion Eng. Design 81 (2006), 1957

[9] H. Laqua, H. Niedermeyer, J. Schacht et al., Real-time software for the fusion experiment WENDELSTEIN 7-X, Fusion Eng. Design 81 (2006), 1807

[10] P. Heimann, S. Heinzel, Ch. Hennig, H. Kroiss, G. Kühner, H. Kühntopf, J. Maier, J. Reetz, M. Zilker, "The data acquistion system of W7X”, Proceedings of the 3rd IAEA Technical Commitee Meeting on 
Steady-State Operation of Magnetic Fusion Devices, 2-3 May 2002, Greifswald, Germany, 6-7 May 2002, Arles, France.

[11] G. Kühner, P. Heimann, S. Heinzel et al., Editor for system configuration and experiment program specification, 2004, Fusion Eng. Design 71 (2004), 225

[12] H. Kühntopf, Kross, H. , T. Bluhm, T. et al., "Specialized editor for processing objects in a database to prepare discharges for WENDELSTEIN 7-X”, Fusion Eng. Design 81 (2006), 1741 